\title{
The importance for growth of dietary intake of calcium and vitamin D
}

\section{A importância do consumo dietético de cálcio e vitamina $D$ no crescimento}

\section{Aline L. Bueno' ${ }^{1}$, Mauro A. Czepielewski²}

\section{Resumo}

Objetivo: Verificar o papel nutricional do cálcio e da vitamina $D$ no processo de crescimento e desenvolvimento infanto-juvenil, visando, em especial, a prevenção e o tratamento do atraso de crescimento causado por deficiência nutricional.

Fontes dos dados: As informações foram coletadas a partir de artigos publicados nas 2 últimas décadas, pesquisados nas bases de dados SciELO, PubMed e MEDLINE, livros técnicos e publicações de organizações internacionais.

Síntese dos dados: O crescimento sofre influência de fatores intrínsecos (genéticos e metabólicos) e extrínsecos (fatores ambientais, como alimentação, saúde, higiene, habitação, e o acesso aos serviços de saúde). Entre os fatores nutricionais, destacam-se as deficiências de vitaminas e oligoelementos que podem se associar à desnutrição ou depender da absorção insuficiente dos mesmos. Sendo o cálcio um dos principais componentes do tecido mineral ósseo, este é essencial para uma adequada formação óssea e, considerando que a vitamina $\mathrm{D}$ desempenha papel importante no metabolismo do cálcio, uma dieta insuficiente nesses nutrientes pode influenciar a formação do esqueleto e o processo de crescimento e desenvolvimento.

Conclusões: A baixa ingestão ou baixa absorção de cálcio e vitamina $D$ em crianças e adolescentes pode limitar seu desenvolvimento estatural, sendo necessário fornecer quantidades suficientes de ambos na fase crítica do crescimento.

J Pediatr (Rio J). 2008;84(5):386-394: Transtornos do crescimento, raquitismo, ingestão de alimentos, recomendações nutricionais.

\section{Introdução}

O crescimento somático normal é um processo complexo determinado por fatores celulares, interação genética e também por fatores externos, como atividade física, infecções, aspectos psicossociais e econômicos, doenças crônicas, fatores metabólicos e hormonais e, finalmente, alimentação ${ }^{1}$. De modo geral, todo indivíduo nasce com um potencial genético de crescimento, que poderá ou não ser atingido, dependendo das condições de vida a que esteja submetido. Assim, pode-se dizer que a altura final é o resultado da interação entre sua

\begin{abstract}
Objective: To investigate the role of dietary calcium and vitamin $D$ in the process of growth and development of children and adolescents, focusing in particular on the prevention and treatment of delayed growth caused by nutritional deficiency.
\end{abstract}

Sources: Information was gathered from articles published in the last 2 decades, from searches on the databases SciELO, PubMed and Medline, technical books and publications of international organizations.

Summary of the findings: Growth is influenced by intrinsic (genetic and metabolic) and extrinsic factors (environmental factors such as diet, health, hygiene, housing and access to health services). Among the nutritional factors are vitamin and oligoelement deficiencies which may be associated with malnutrition or caused by insufficient absorption. Since calcium is one of the main mineral components of bone tissue, it is essential for adequate bone formation and, considering that vitamin $D$ plays an important role in calcium metabolism, a diet with insufficient quantities of these nutrients can impact on the formation of the skeleton and on the process of growth and development.

Conclusions: In children and adolescents, low intake or low absorption of calcium and vitamin D may limit their statural growth, and it is necessary to supply sufficient quantities of both during the critical growth phases.

J Pediatr (Rio J). 2008;84(5):386-394: Growth disorders, rickets, nutrition, diet, nutritional recommendations.

carga genética e os fatores do meio ambiente que permitirão a maior ou menor expressão do seu potencial genético ${ }^{2,3}$.

O crescimento deficiente pode manifestar-se clinicamente como estatura abaixo do esperado para o potencial familiar, estatura abaixo do esperado para a população geral ou por velocidade de crescimento inferior à esperada, considerando o sexo, a idade cronológica e o estágio puberal da criança. Portanto, a criança com baixa estatura, por definição, é aquela que se encontra abaixo do percentil 3, ou seja, 2 desvios padrão nos gráficos de crescimento para a média de estatura da população geral ${ }^{4,5}$.

1. Nutricionista. Mestre, Universidade Federal do Rio Grande do Sul (UFRGS), Porto Alegre, RS

2. Médico. Doutor, Universidade Federal de São Paulo - Escola Paulista de Medicina (UNIFESP-EPM), São Paulo, SP. Professor associado, Programa de PósGraduação em Ciências Médicas: Endocrinologia e Nutrição, Faculdade de Medicina, UFRGS, Porto Alegre, RS.

Não foram declarados conflitos de interesse associados à publicação deste artigo.

Como citar este artigo: Bueno AL, Czepielewski MA. The importance for growth of dietary intake of calcium and vitamin D. J Pediatr (Rio J). 2008;84(5): 386-394.

Artigo submetido em 06.05.08, aceito em 14.05.08.

doi:10.2223/JPED.1816 
Tabela 1 - Fatores dietéticos que afetam o balanço de cálcio

\begin{tabular}{|c|c|c|}
\hline & Diminui & Aumenta \\
\hline \multirow[t]{7}{*}{ Absorção } & Fibra & Alimentação \\
\hline & Fitato & Lactose \\
\hline & Oxalato & Carboidratos \\
\hline & Cafeína & Lisina \\
\hline & Gordura & Gordura \\
\hline & Fósforo & - \\
\hline & Ferro & - \\
\hline \multirow[t]{4}{*}{ Excreção } & Fósforo & Proteína \\
\hline & Cinza alcalina & Sódio \\
\hline & - & Cloreto \\
\hline & - & Cinza ácida \\
\hline
\end{tabular}

Então, como o crescimento normal depende da interação entre vários fatores, a baixa estatura pode ser resultante de diversas causas, entre elas as causas genéticas, endócrinas, secundárias a doenças crônicas e as causas nutricionais.

Infecções e consumo alimentar inadequado estão bem estabelecidos como causas de baixa estatura ${ }^{6,7}$. Contudo, a possibilidade da deficiência de algum micronutriente ter algum papel na etiologia do retardo de crescimento tem despertado atenção recentemente. Isso porque alguns micronutrientes são requisitos para promoção do crescimento físico, para a maturação sexual, para o desenvolvimento neuromotor e para a integridade e o funcionamento do sistema imune. Assim, o completo potencial genético de uma criança para o crescimento físico e desenvolvimento mental pode também ser comprometido devido a deficiências subclínicas de micronutrientes ${ }^{8}$.

Como causa dietética de baixa estatura caracterizada pela deficiência de um micronutriente, destacamos o raquitismo causado pela deficiência de vitamina $D$, doença que resulta em retardo de crescimento, fraqueza muscular, deformidade esquelética, hipocalcemia e tetania. Epidemia no século XIX foi quase completamente erradicada com o encorajamento da exposição solar e com a fortificação do leite em vitamina D. Mas a deficiência desta vitamina voltou a ser uma epidemia entre crianças, e o raquitismo tornou-se assunto de saúde em todo o mundo. Além da deficiência de vitamina $D$, a deficiência de cálcio causa raquitismo ${ }^{9}$.

Contudo, acreditamos que, mesmo antes do desenvolvimento do raquitismo, a deficiência dietética de cálcio e vitamina $D$ pode prejudicar o crescimento e desenvolvimento. Assim, esta revisão sumariza o papel do cálcio e da vitamina
D e sua importância na manutenção da saúde geral, crescimento e desenvolvimento de crianças e adolescentes.

\section{Cálcio}

O cálcio é um elemento fundamental ao organismo, e sua importância está relacionada às funções que desempenha na mineralização óssea, principalmente na saúde óssea, desde a formação, manutenção da estrutura e rigidez do esqueleto ${ }^{10,11}$.

O cálcio absorvido da dieta depende do balanço entre a ingestão, a absorção (ingestão menos a perda fecal) e a excreção. Vários fatores influenciam estes mecanismos, conforme apresentado na Tabela $1^{11,12}$.

\section{Recomendações nutricionais de cálcio}

As recomendações nutricionais de cálcio variam durante a vida dos indivíduos, com maiores necessidades durante períodos de rápido crescimento, como na infância e na adolescência, durante a gravidez e lactação, na deficiência de cálcio, na prática de exercícios que resultem em alta densidade óssea e aumentam a absorção de cálcio e na velhice ${ }^{13}$. A ingestão ideal de cálcio é aquela que conduza a um pico de massa óssea adequado na criança e adolescente, mantenha-o no adulto e minimize a perda na senilidade ${ }^{14}$.

O Standing Commitee on the Scientific Evaluation of Dietary Reference Intakes, o Food and Nutrition Boarde o Institute of Medicine - National Academy Science estabeleceram recomendações dietéticas para cálcio em vários grupos etários. Os requerimentos de cálcio foram estabelecidos baseados em três indicadores: risco de fratura, medidores de massa muscular e retenção máxima de cálcio ${ }^{12,15}$ (Tabela 2). 
Tabela 2 - Recomendação nutricional para cálcio - Dietary Reference Intake ${ }^{15}$

$\begin{array}{ll}\text { Faixa etária* } & \text { AI (mg/dia) }\end{array}$

Infância

0 a 6 meses

Adolescentes

9 a 13 anos

14 a 18 anos

Adultos

Gestação

Lactação

AI = consumo adequado. É a estimação determinada experimentalmente do consumo de nutrientes por grupos definidos de pessoas saudáveis. A AI é utilizada se investigações cientificas não são suficientes para estabelecer o requerimento médio estimado (EAR). Avalia a prevalência de consumo inadequado em um grupo. Para lactentes saudáveis alimentados com leite materno, a AI é um consumo médio estimado.

* Todos os grupos, exceto gestação e lactação, são masculino e feminino.

Todavia, apesar do acordo entre os países desenvolvidos quanto às recomendações dietéticas de cálcio, existem algumas dúvidas quanto à sua aplicação em países em desenvolvimento, como o Brasil, pois todas as tabelas são baseadas em dados sobre população branca de países desenvolvidos, desconsiderando as diferenças de etnia, hábitos culturais e alimentares e geográficas observadas nos países ${ }^{10}$. Salientamos, assim, a necessidade de desenvolver recomendações dietéticas específicas para nossa população, considerando as variações regionais tão diversas do nosso país.

Por não ser produzido endogenamente, o cálcio é somente adquirido através da ingestão diária de alimentos que o contenham ${ }^{14}$. Como alimentos ricos em cálcio, destacam-se o leite e seus derivados (iogurte e queijo) com baixo teor de gorduras $^{16}$.

A alta biodisponibilidade do cálcio nos produtos lácteos está relacionada com o conteúdo de vitamina $\mathrm{D}$ e com a presença de lactose, que aumentam a sua absorção no intestino ${ }^{17}$. Além disso, como o pH do leite é alcalino, o cálcio se mantém em suspensão pela formação de caseinato de cálcio, de citrato de cálcio e de um complexo com a lactose. Assim, a lactose, caseinato e citrato presentes no leite e derivados parecem explicar a melhor absorção de cálcio destas fontes em relação a outras ${ }^{14}$. Apesar dos queijos conterem pouca lactose, o cálcio está prontamente disponível neste alimento $^{10}$. 
Tabela 3 - Fontes alimentares ricas em cálcio

\begin{tabular}{|c|c|c|}
\hline Alimento & Porção & Cálcio (mg) \\
\hline Leite integral & 1 xícara de chá & 290 \\
\hline Leite semidesnatado & 1 xícara de chá & 297 \\
\hline Leite desnatado & 1 xícara de chá & 302 \\
\hline Queijo branco & Fatia $(30 \mathrm{~g})$ & 205 \\
\hline Queijo mozarela & Fatia $(20 \mathrm{~g})$ & 120 \\
\hline Requeijão & Colher de sopa rasa (15 g) & 84,75 \\
\hline Iogurte natural & Pote $(200 \mathrm{~g})$ & 228 \\
\hline Coalhada & Pote $(200 \mathrm{~g})$ & 130 \\
\hline Sardinha em conserva & $100 \mathrm{~g}$ & 402 \\
\hline Peixe & Filé ou posta & $50-60$ \\
\hline Feijão de soja cozido & 1 xícara & 175 \\
\hline Brócolis cozido & $100 \mathrm{~g}$ & 113 \\
\hline Couve cozida & 1 xícara & 148 \\
\hline Repolho picado & 1 xícara & 94 \\
\hline Batata assada & 1 unidade média & 115 \\
\hline Laranja & 1 unidade & 96 \\
\hline Mamão & 1 unidade média & 62 \\
\hline Melancia & 1 fatia grande & 22 \\
\hline
\end{tabular}

Recentemente, com o diagnóstico mais freqüente de intolerância a lactose, esta situação exige cuidado especial na manutenção da ingestão adequada de cálcio nestes pacientes. Esta hipótese é reforçada pelos resultados de Medei$\operatorname{ros}^{17}$, que encontraram menor ingestão de cálcio $(p<0,001)$ entre crianças que consumiam dieta isenta de leite de vaca e derivados ${ }^{18}$.

Entretanto, não somente o consumo de leite e derivados contribui para a ingestão de cálcio dos indivíduos. São fontes de cálcio vegetais de folhas verdes escuras, tais como couve, couve-manteiga, folhas de mostarda, de brócolis e de nabo, mas o cálcio está pouco biodisponível nesses alimentos. Sardinha, moluscos bivalves, ostras, salmão e leguminosas, como a soja, também contêm cálcio em quantidades descritas na Tabela $3^{10,19,20}$.

\section{Consumo dietético de cálcio}

Até recentemente, não se acreditava que o baixo consumo de cálcio resultasse em prejuízos à saúde. Atualmente se considera que variações mundiais na prevalência da deficiência de cálcio podem influenciar a distribuição óssea e os hábitos alimentares nas diferentes populações, em decorrência de diferenças genéticas, étnicas, geográficas (latitudes), e relacionadas a fatores culturais e estilo de vida ${ }^{11}$.

Podemos citar alguns estudos que demonstram baixo consumo de alimentos que contenham cálcio, especialmente em idade de desenvolvimento físico, fato que pode futuramente causar déficit de crescimento ou até doenças ósseas.

Rajeshwari et al. acompanharam crianças dos 10 anos até a vida adulta, demonstrando que o consumo de cálcio está diminuído durante este período, apesar do aumento do consumo energético. Além disso, verificou que há diminuição considerável no consumo total de cálcio na infância ( $54 \%$ abaixo da recomendação) à idade adulta (77\% abaixo da recomendação) ${ }^{21}$.

Em outro estudo, Salamoun et al. avaliaram o consumo de cálcio e vitamina $D$ entre crianças e adolescentes de países do mediterrâneo e encontraram consumo subótimo de ambos os nutrientes (consumo médio de cálcio de $816 \pm 776,8$ $\mathrm{mg} /$ dia e de vitamina D de $129 \pm 116,1 \mathrm{UI} /$ dia). Apenas $12 \%$ 
Tabela 4 - Recomendação nutricional para Vitamina D - Dietary Reference Intake 15

\begin{tabular}{|c|c|}
\hline Faixa etária* & AI ( $\mu g / d i a)^{+\neq}$ \\
\hline Infância (0 a 12 meses) & 5 \\
\hline Crianças ( 1 a 8 anos) & 5 \\
\hline Adolescentes & 5 \\
\hline \multicolumn{2}{|l|}{ Adultos } \\
\hline 19 a 50 anos & 5 \\
\hline 51 a 70 anos & 10 \\
\hline$>70$ anos & 15 \\
\hline Gestação & 5 \\
\hline Lactação & 5 \\
\hline
\end{tabular}

* Todos os grupos, exceto gestação e lactação, são masculino e feminino. Recomendado pelo Institute of Medicine, National Academy of Sciences.

+ Como colecalciferol. $1 \mu \mathrm{g}$ de colecalciferol = 40 UI de vitamina D.

* $\mathrm{Na}$ falta de exposição solar adequada.

atingiram o consumo diário adequado de cálcio, e 16\% de vitamina $D^{22}$

No Brasil, Lerner et al. avaliaram o consumo de cálcio em adolescentes de escolas públicas de Osasco (SP) e encontraram que o consumo médio diário de cálcio não foi significativamente diferente entre meninos e meninas, estando, nos dois casos, perto de $50 \%$ do recomendado (média de ingestão de cálcio de $628,85 \pm 353,82 \mathrm{mg} /$ dia entre os meninos e $565,68 \pm 295,43 \mathrm{mg} / \mathrm{dia}$ entre as meninas). Somente $6,2 \%$ dos meninos e $2,8 \%$ das meninas apresentaram consumo adequado em cálcio, à semelhança de outros estudos realizados no exterior ${ }^{21-23}$.

\section{Vitamina D}

A $1,25-(\mathrm{OH})_{2} \mathrm{D}_{3}$ é um hormônio que regula o metabolismo do cálcio e do fósforo. Assim sendo, sua principal função é manter os níveis séricos de cálcio e fósforo em um estado normal capaz de propiciar condições à maioria das funções metabólicas, entre elas a mineralização óssea9,16. Por estar envolvida no crescimento esquelético, a vitamina $D$ torna-se essencial durante a infância e a adolescência ${ }^{24}$.

Níveis séricos normais de vitamina $\mathrm{D}$ promovem a absorção de $30 \%$ do cálcio dietético e mais de $60-80 \%$ em períodos de crescimento, devido à alta demanda de cálcio ${ }^{25}$. Por isso, durante a infância, a deficiência de vitamina $D$ pode causar retardo de crescimento, anormalidades ósseas, aumentando o risco de fraturas na vida adulta ${ }^{26}$.

\section{Recomendações nutricionais de vitamina $D$}

As recomendações nutricionais diárias de vitamina D são difíceis de estabelecer com exatidão, pois ela é produzida endogenamente e depositada no tecido adiposo por longos períodos de tempo, e suas necessidades também dependem do consumo dietético de cálcio e fósforo, idade, sexo, pigmentação da pele e exposição solar. Historicamente se definiu como quantidade de vitamina $D$ suficiente para prevenir o raquitismo uma colher de sopa de óleo de peixe. Ainda hoje não existem evidências suficientes para estabelecer sua recomendação, mas seu consumo adequado diário foi estabelecido $^{26,27,15}$ (Tabela 4).

A pele tem alta capacidade de sintetizar vitamina $D$, pois a exposição solar que causa leve eritema na pele em crianças e adultos vestindo trajes de banho é estimada como sendo igual a 15 vezes a recomendação diária de vitamina $D$, e a exposição a um eritema leve em $6 \%$ do corpo é igual a um consumo de 15-25 $\mu$ g de colecalciferol 26,28 .

Ainda assim é difícil determinar a quantidade de exposição solar (superfície total de pele exposta em um determinado tempo) necessária para prevenir a deficiência de vitamina $\mathrm{D}$ e o raquitismo na infância. Além disso, há uma preocupação crescente quanto à exposição UVB nesta fase e sua relação com o câncer de pele na vida adulta, pois existe uma correlação positiva entre a ocorrência de melanoma maligno entre adultos e a quantidade de exposição solar na infância ${ }^{29}$.

Apesar de a vitamina D ser produzida por exposição da pele aos raios solares, seu consumo dietético se torna essencial quando a exposição solar é insuficiente para alcançar as necessidades diárias. Isso tem se tornado comum, particularmente entre pessoas residentes em centros urbanos que estão expostos a níveis subótimos de raios solares $27,30,31$ (Tabela 5).

Entretanto, são escassos os alimentos fonte de vitamina D, como a gema de ovo, fígado, manteiga e leite. Além disso, 
Tabela 5 - Fatores de risco para hipovitaminose $D^{30}$

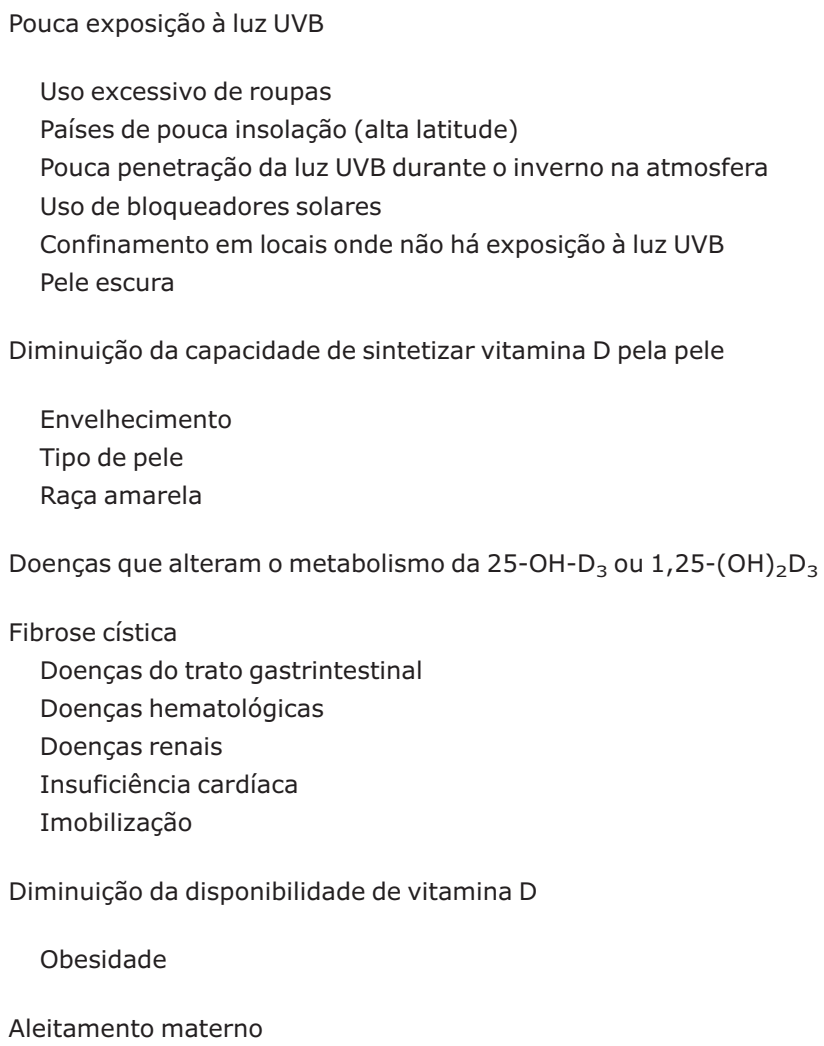

estes alimentos são atualmente pouco consumidos em função do elevado conteúdo de colesterol ${ }^{32}$. De modo geral, carnes e peixes magros têm apenas traços desta vitamina, estando as maiores concentrações presentes no arenque e na cavala ${ }^{16}$. Óleos de fígado de peixes como atum e linguado, bacalhau em particular e peixes como salmão, cavala, sardinha, enguia, arenque e atum são ricos em vitamina $D^{19,33}$ (Tabela 6).

Outra fonte seriam os cogumelos, que naturalmente possuem pequenas quantidades de vitamina $D$, mas este alimento também não é freqüentemente consumido e está sujeito a grande variação sazonal no seu conteúdo de vitamina $D^{32}$.

Infelizmente, a literatura é escassa em trabalhos envolvendo biodisponibilidade dessa vitamina. Alguns fatores dietéticos têm sido apontados como auxiliares ou redutores da biodisponibilidade da vitamina $\mathrm{D}$. Porém, sabe-se que o leite ingerido conjuntamente com fontes naturais de vitamina $D$ pode elevar de três a 10 vezes sua absorção, fato que pode ser explicado pela presença da lactoalbumina. Ácidos graxos de cadeia longa também facilitam a absorção de vitamina $D$, quando comparada a doses farmacológicas dessa vitamina. Já a ingestão de etanol e de fibras leva à diminuição da sua biodisponibilidade, pois promove perda biliar ${ }^{34}$.
Assim, a irradiação solar pode garantir o aporte de vitamina D na infância, mas com aumento do risco de câncer de pele; além disso, fontes alimentares desta vitamina são restritas. Sendo assim, uma opção mais segura de alcançar as necessidades dietéticas durante o crescimento seria o uso de suplementos dietéticos do tipo farmacêuticos ou o enriquecimento de alimentos com vitamina $D^{27,35}$.

\section{Consumo dietético de vitamina $D$}

Estima-se que uma dieta saudável seja suficiente para alcançar plenitude em vitamina $D$, porém, como vimos antes, isso nem sempre acontece. Esta é a razão pela qual a deficiência de vitamina $D$ tem se tornado freqüente em crianças e adolescentes em fase de crescimento nos EUA e Europa ${ }^{27,25}$.

Docio et al. realizaram um estudo para determinar níveis desejáveis de $25-\mathrm{OH}-\mathrm{D}_{3}$ em crianças e saber se elas mantêm estes níveis durante todo ano e verificaram que o limite mínimo para os níveis de $25-\mathrm{OH}-\mathrm{D}_{3}$ desejado em crianças é algo entre 12 e $20 \mathrm{ng} / \mathrm{mL}$. Contudo, $31 \%$ das 51 crianças normais estudadas no inverno apresentaram níveis inferiores a $12 \mathrm{ng} / \mathrm{mL}$, e $80 \%$ tiveram níveis abaixo de $20 \mathrm{ng} / \mathrm{mL}$. Quando analisaram a dieta, encontraram consumo médio de $790 \pm 156$ $\mathrm{mg} /$ dia de cálcio e $160 \pm 80 \mathrm{IU} /$ dia de vitamina $\mathrm{D}$, ambos abaixo do recomendado ${ }^{36}$.

Gordon et al. realizaram estudo em hospital urbano de Boston, no qual foi avaliada a prevalência de deficiência de 
Tabela 6 - Alimentos ricos em vitamina $D^{33}$

\begin{tabular}{lcc}
\hline Alimento & Medidas caseiras $/ \mathbf{g}$ & $\mu \mathrm{g}$ \\
\hline Atum & 2 médios $(90 \mathrm{~g})$ & 3,68 \\
Sardinha crua & $100 \mathrm{~g}$ & 17 \\
Sardinha enlatada & $100 \mathrm{~g}$ & 40,3 \\
Óleo de peixe & 1 colher de sopa & 0,45 \\
Manteiga & 1 colher de sopa cheia & 1,12 \\
Fígado de boi & $100 \mathrm{~g}$ & 1,25 \\
Fígado de frango & $100 \mathrm{~g}$ & 0,53 \\
Gema de ovo fresca & $100 \mathrm{~g}$ & 0,875 \\
Ovo de galinha fresco & $100 \mathrm{~g}$ & 0,17 \\
Leite integral & 1 copo $(240 \mathrm{~mL})$ & 0,62 \\
Cogumelos & $100 \mathrm{~g}$ &
\end{tabular}

vitamina D sérica em 307 adolescentes saudáveis. Foram encontrados $24,1 \%$ de pacientes deficientes em vitamina $D$, dos quais 4,6\% estavam severamente deficientes. Estação do ano (inverno e primavera), etnia (afro-americanos), consumo de leite, índice de massa corporal (IMC) e atividade física foram fatores independentes e significativos de hipovitaminose $D^{24}$.

Estudos com pré-adolescentes também demonstram deficiência nos níveis séricos e consumo de vitamina D. Rajakumar et al. avaliaram a proporção de deficiência de vitamina $D$ em pré-adolescentes estadunidenses afro-descendentes de 6 a 10 anos. Quarenta e nove por cento apresentaram níveis séricos de $25-\mathrm{OH}-\mathrm{D}_{3}$ insuficientes $(24 \pm 10,5 \mathrm{ng} / \mathrm{mL})$. O consumo médio de vitamina $D$ foi de $277 \pm 146$ IU/dia, e $39 \%$ deles não atingiram o consumo diário adequado de vitamina $D^{37}$.

No Brasil, até o presente momento, existem poucos estudos sobre prevalência de hipovitaminose D. O Rio Grande do Sul, devido às suas características climáticas, apresenta maior possibilidade de deficiência desta. Em um estudo realizado no Hospital de Clínicas de Porto Alegre (HCPA), Premaor \& Furlanetto encontraram baixos níveis séricos de $25-\mathrm{OH}-\mathrm{D}_{3}$ nos pacientes internados nas equipes de medicina interna. Todavia, estes pacientes eram adultos e apresentavam vários fatores de risco para desenvolver a doença, portanto ainda não se conhece a extensão real deste distúrbio em nosso meio ${ }^{30}$.

\section{Cálcio e vitamina D dietéticos no crescimento}

Sendo assim, o decréscimo do consumo de cálcio e vitamina $D$ em períodos de crescimento pode influenciar negativamente o desenvolvimento ósseo, causando não apenas raquitismo, que é o resultado final da deficiência de vitamina
D, como também prejudicando o alcance da altura programada geneticamente ${ }^{25}$.

As necessidades de cálcio durante a puberdade e adolescência são maiores do que em qualquer outro período da vida, em função do acelerado desenvolvimento muscular, esquelético e endócrino ${ }^{11}$. O depósito mineral ósseo durante o crescimento puberal parece depender da absorção dietética de cálcio, assim como da redução da sua excreção, e isso depende de um adequado estado de vitamina D. Apesar disso, o entendimento da relação entre absorção de cálcio e vitamina $D$ e crescimento é limitado ${ }^{38}$.

Abrams et al. compararam a altura de 315 meninas entre 5 e 15 anos com a absorção de cálcio dietético e encontraram relações positivas $(r=0,18 ; p=0,001)$, demonstrando que um aumento na eficiência absortiva é, em parte, regulado para alcançar as necessidades do tamanho esquelético final. No entanto, esta relação permanece incerta, podendo ser devida a componentes genéticos ou a diferenças étnicas. Outra hipótese seria que a absorção de cálcio estivesse diretamente relacionada com a maior superfície intestinal dos indivíduos mais altos. Estes achados dependeram do estágio puberal independente da altura das meninas, pois foi observado um aumento na absorção de cálcio durante o início da puberdade ${ }^{38}$.

Prentice et al. avaliaram o efeito da suplementação com carbonato de cálcio ( $1.000 \mathrm{mg} /$ dia) versus placebo em 143 meninos, entre 16 e 18 anos de idade, durante 13 meses, quanto à aquisição óssea e crescimento ósseo. A intervenção resultou em melhora no conteúdo mineral ósseo total, que aumentou $1,3 \%(p=0,02)$, associada a incremento na altura de $0,4 \%(p=0,0004)$ equivalente a $7 \mathrm{~mm}$. Essa associação 
não foi encontrada em estudo com crianças menores e meninas na mesma faixa etária ${ }^{39}$. Apesar de existirem estudos com suplementação de cálcio, estudos relacionando cálcio dietético e crescimento são escassos, assim como a vitamina D.

Os resultados de Black et al. confirmaram a visão de que crianças com longa história de baixo consumo de leite têm baixa ingestão de cálcio dietético ( $443 \pm 230 \mathrm{mg} / \mathrm{dia})$ e pobre saúde óssea (conteúdo mineral ósseo 0,45 g menor; $\mathrm{p}<0,01$ ) em comparação com crianças que consomem leite. Isso também reforça a hipótese de que crianças que não consomem leite de vaca têm estatura menor que aquelas que consomem leite regularmente $(0,65 \mathrm{~cm}$ menores; $p<0,01)$. Além desses resultados, o escore z para conteúdo mineral ósseo total correlacionou-se positivamente com o consumo dietético de cálcio $(r=0,38 ; p<0,006)$. Neste estudo, o consumo dietético de cálcio das crianças que não consomem leite não alcançou a grande quantidade de cálcio necessária para demanda de crescimento puberal ${ }^{40}$.

\section{Conclusões}

Assim, podemos concluir que, durante o crescimento, o suprimento adequado de cálcio e vitamina $D$ é considerado criticamente importante no desenvolvimento ósseo e, se a criança está apta a alcançar seu potencial genético de crescimento e pico de massa óssea, a dieta deve ter quantidade suficiente destes nutrientes. Também ficou claro o baixo consumo dietético de cálcio e vitamina $D$ entre crianças e adolescentes, e que este causa efeito deletério na saúde esquelética e metabolismo ósseo. É necessário investigar as causas da baixa ingestão de cálcio e vitamina $D$ entre indivíduos no período de crescimento, como na infância e na adolescência, estabelecer estratégias nutricionais para aumentar seu consumo dietético e propiciar o alcance de alimentos ricos nestes nutrientes entre as populações em risco nutricional.

\section{Agradecimentos}

Agradecemos aos funcionários, residentes e pósgraduandos do Ambulatório de Baixa Estatura do Serviço de Endocrinologia do HCPA pelo seu auxílio em diversas fases de nosso trabalho. Também somos gratos ao Fundo de Incentivo à Pesquisa e Eventos (FIPE) do HCPA, à Coordenação de Aperfeiçoamento de Pessoal de Nível Superior (CAPES) e ao Centro de Endocrinologia e Diabetes do Rio Grande do Sul (CEDERS) pelo suporte financeiro para o desenvolvimento de vários projetos.

\section{Referências}

1. Liberman B, Cukiert A. Fisiologia e fisiopatologia do hormônio do crescimento. São Paulo: Lemos Editorial; 2004.

2. Hall R, Anderson J, Stuart GA, Besser GM. Clinical Endocrinology. 2nd ed. 1994.

3. Giugliani ER. Baixa estatura: um mal da sociedade brasileira. J Pediatr (Rio J). 1994;70:261.

4. Larsen PR, Kronenberg $H$, Melmed S, et. al. Willians textbook of endocrinology, 10ed. Phyladelphia.: Elsevier.; 2003.
5. Cowell CT. Short stature. In: Brook CG, Hindmarsh PC, editors. Clinical pediatric endocrinology. 3th ed. Cambridge: Blackwell Science; 1996. p. 136-72.

6. Onis M, Blössner M. The World Health Organization Global Database on Child Growth and Malnutrition: methodology and applications. Int J Epidemiol. 2003;32:518-26.

7. Aerts $D$, Drachler ML, Giugliani ER. Determinants of growth retardation in Southern Brazil. Cad Saude Publica. 2004; 20:1182-90.

8. Singh M. Role of micronutrients for physical growth and mental development. Indian J Pediatr. 2004;71:59-62.

9. Holick MF. Resurrection of Vitamin D deficiency and rickets. J Clin Invest. 2006; 116:2062-72.

10. Cobayashi F. Cálcio: seu papel na nutrição e saúde. Compacta Nutr. 2004;2:3-18.

11. Joint FAO/WHO Expert Consultation on Human vitamin and mineral requirements. Bangkok; 1998.

12. Branca F, Valtueña S. Calcium, physical activity and bone health - building bones for a stronger future. Public Health Nutr. 2001; 4:117-23.

13. Flynn A. The role of dietary calcium in bone health. Proc Nutr Soc. 2003;62:851-8.

14. Grüdtner VS, Weingrill P, Fernandes AL. Aspectos da absorção no metabolismo do cálcio e vitamina D. Rev. Bras. Reumatol. 1997; 37:143-51.

15. Food and Nutrition Board and Institute of Medicine. Dietary reference intakes for calcium, phosphorus, magnesium, vitamin D, and fluoride. Washington, DC: National Academy Press; 2002.

16. Lopez FA, Brasil AD. Nutrição e dietética em clínica pediátrica. São Paulo: Atheneu; 2004.

17. Medeiros LC, Speridião PG, Sdepanian VL, Fagundes-Neto U, Morais MB. Ingestão de nutrientes e estado nutricional de crianças em dieta isenta de leite de vaca e derivados. J Pediatr (Rio J). 2004;80:363-70.

18. Vitolo MR. Nutrição: da gestação à adolescência. Rio de Janeiro: Reichmann \& Affonso; 2003.

19. Tabela brasileira de composição de alimentos/NEPA-UNICAMP. $2 a$ ed. Campinas, SP: NEPA-UNICAMP; 2006.

20. Pinheiro AB, Lacerda SE, Benzecry EH. Tabela para avaliação de consumo alimentar em medidas caseiras. $4 a$ ed. São Paulo: Atheneu; 2002.

21. Rajeshwari R, Nicklas TA, Yang SJ, Berenson GS. Longitudinal changes in intake and food sources of calcium from childhood to young adulthood: The Bogalusa Heart Study. J Am Coll Nutr. 2004;23:341-50.

22. Salamoun MM, Kizirian AS, Tannous RI, Nabulsi MM, Choucair MK, Deeb ME, et al. Low calcium and vitamin D intake in healthy children and adolescents and their correlates. Eur J Clin Nutr. 2005; 59:177-84.

23. Lerner BR, Lei DL, Chaves SP, Freire RD. O cálcio consumido por adolescentes de escolas públicas de Osasco, São Paulo. Rev Nutr. 2000; 13:57-63.

24. Gordon CM, DePeter KC, Feldman HA, Grace E, Emans SJ. Prevalence of vitamin $D$ deficiency among healthy adolescents. Arch Pediatr Adolesc Med. 2004;158:531-7.

25. Holick MF. Sunlight and vitamin D for bone health and prevention of autoimmune diseases, cancers and cardiovascular disease. Am J Clin Nutr. 2004;80:1678S-88S. 
26. Holick MF. Vitamin D deficiency. N Engl J Med. 2007;357:266-81.

27. Hochberg Z. Vitamin D and rickets. Consensus development for the supplementation of vitamin $D$ in childhood and adolescence. Endocr Dev Basel. 2003;6:259-81.

28. Molgaart C, Michaelsen KF. Vitamin D and bone health in early life. Proc Nutr Soc. 2003;62:823-8.

29. Greer FR. Issues in establishing vitamin $D$ recommendations for infants and children. Am J Clin Nutr. 2004;80:1759S-62S.

30. Premaor MO, Furlanetto TW. Hipovitaminose D em adultos: entendendo melhor a apresentação de uma velha doença. Arq Bras Endocrinol Metab. 2006;50:25-37.

31. Ladhani S, Srinivasan L, Buchanan C, Allgrove J. Presentation of vitamin D deficiency. Arch Dis Child. 2004;89:781-4

32. Calvo MS, Whiting SJ. Public Health Strategies to overcome barriers to optimal vitamin D status in population with special needs. J Nutr. 2006;136:1135-9.

33. United States Department of Agriculture. USDA National Nutrient Database for Standard Reference, Release 19. http:// www.ars.usda.gov/nutrientdata. Access: 02.09.2007.

34. Mourão DM, Sales NS, Coelho SB, Pinheiro-Santana HM. Biodisponibilidade de vitaminas lipossolúveis. Rev Nutr. 2005; 18:529-39.

35. Newmark HL, Heaney RP, Lachance PA. Should calcium and vitamin $D$ added to the current enrichment program for cereal-grain products? Am J Clin Nutr. 2004; 80:264-70.
36. Docio S, Riancho JA, Pérez A, Olmos JM, Amado JA, González-macías J. Seasonal deficiency of vitamin D in children: a potential target for osteoporosis-preventing strategies? J Bone miner Res. 1998;13:544-8.

37. Rajakumar K, Fernstrom JD, Janosky JE, Greenspan SL. Vitamin D Insufficiency in Preadolescent African-American Children. Clin Pediatr (Phila). 2005;44:683-92.

38. Abrams AS, Griffin IJ, Hawthorne KM, Liang L. Height and height Z-score are related to calcium absorption in five to fifteen year-old girls. J Clin Endocrinol Metab. 2005;90:5077-81.

39. Prentice A, Ginty F, Stear SJ, Jones SC, Laskey MA, Cole TJ. Calcium suplementation increases stature and bone mineral mass of 16- to 18-year-old boys. J Clin Endocrinol Metab. 2005; $90: 3153-61$.

40. Black RE, Williams SM, Jones IE, Goulding A. Children who avoid drinking cow milk have low dietary calcium intakes and poor bone health. Am J Clin Nutr. 2002;76:675-80.

Correspondência:

Mauro A. Czepielewski

Serviço de Endocrinologia

Hospital de Clínicas de Porto Alegre

Rua Ramiro Barcelos, 2350 - prédio 12/40 andar

CEP 90035-003 - Porto Alegre, RS

Tel.: (51) 3316.5600

E-mail: maurocze@terra.com.br 